Research Paper

\title{
miR-186-5p Promotes Apoptosis by Targeting IGF-1 in SH-SY5Y OGD/R Model
}

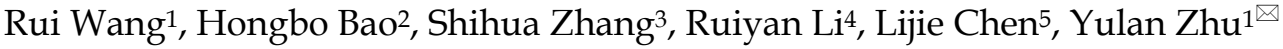 \\ 1. Department of Neurology, the Second Affiliated Hospital of Harbin Medical University, Harbin, Heilongiiang Province, 150001, China \\ 2. Department of Neurosurgery, Harbin Medical University Cancer Hospital, Harbin, Heilongiiang Province, 150001, China \\ 3. Department of Neurosurgery, First Affiliated Hospital of Jiamusi University, Jiamusi, Heilongjiang Province, 154002, China. \\ 4. Department of Neurosurgery, the Second Affiliated Hospital of Harbin Medical University, Harbin, Heilongjiang Province, 150001, China \\ 5. Department of Neurology, First Affiliated Hospital of Zhengzhou University, Zhengzhou, Henan Province, 450000, China.
}

$\square$ Corresponding author: Yulan Zhu, Department of Neurology, the Second Affiliated Hospital of Harbin Medical University, Harbin, Heilongjiang Province, 150001, China Email: ylz_yulan@163.com, Tel: +86 0451-86605841

(c) Ivyspring International Publisher. This is an open access article distributed under the terms of the Creative Commons Attribution (CC BY-NC) license (https://creativecommons.org/licenses/by-nc/4.0/). See http://ivyspring.com/terms for full terms and conditions.

Received: 2018.02.04; Accepted: 2018.08.01; Published: 2018.10.19

\begin{abstract}
In recent years, accumulating evidence has revealed that microRNAs play critical roles in ischemia stroke. This study was designed to investigate the expression level and effects of microRNA (miR)-186-5p on ischemia stroke, and its underlying molecular mechanism. Firstly, we demonstrated that miR-186-5p were significantly up-regulated and induced apoptosis in oxygen and glucose deprivation/reperfusion (OGD/R) model. Moreover, we found that miR-186-5p reduced the expression of insulin-like growth factor (IGF)-1, an essential factor for the development of the nervous system. Meanwhile, miR-186-5p inhibitor enhanced cell viability and IGF-1 expression. Furthermore, IGF-1 was confirmed as a direct target gene of miR-186-5p by luciferase activity assay. In addition, miR-186-5p was upregulated in ischemia stroke patients' serum compared with healthy donors. These data demonstrated that miR-186-5p was an adverse factor by inducing neuron apoptosis and suppressing IGF- 1 in ischemia stroke model, and suggested that miR-186-5p may be a diagnostic marker and potential therapeutic target for ischemia stroke patients.
\end{abstract}

Key words: ischemia stroke, miR-186-5p, IGF-1, apoptosis

\section{Introduction}

Stroke is one of the leading causes of death and long-term disability in adult worldwide[1, 2]. Some clinical studies showed that the intravenous thrombolytic therapy within $3 \mathrm{~h}$ from the onset of the symptoms reduced mortality and disability. What happened in the ischemic brain tissue after the blood flowed there again? Which factors played protective roles, and which ones played harmful roles? microRNAs (miRNAs), a class of single-stranded non - coding endogenous small RNA molecules of between 19 and 24 nucleotides in length, play critical roles in a variety of biological processes, including cell proliferation, differentiation, development, cell cycle, metabolism, apoptosis and immunity[3-5]. Accumulative data has demonstrated the important regulatory roles of microRNAs in vascular and neural damage after ischemic stroke[1]. However, the functional significance and mechanisms of miRNAs in cerebrovascular pathophysiology after ischemia stroke remain unclear. miR-186-5p, which is previously reported to serve as a circulating biomarker for certain cancers[6], is a double-edged sword and plays even opposite roles in apoptosis in different physiological and pathological processes. In this study, we investigate miR-186-5p's effect and mechanism in ischemia and reperfusion by inducing apoptosis.

\section{Materials and Methods}

\section{Patients and study design}

The procedure of human serum sample collection was approved by the Ethical Committee of 
Harbin Medical University. Meanwhile, written informed consent was obtained from all healthy donors or acute ischemic stroke patients at the Second Affiliated Hospital of Harbin Medical University. A total of 23 patients (age range, 44-83 years; median age, $65 ; 12$ males and 11 females) confirmed by CT or MRI within 5 days from onset were enrolled between April 2017 and August 2017 and were used as the research subjects. A total of 23 healthy volunteers (age range, 41-78 years; median age, $61 ; 13$ males and 10 females) who underwent physical examination at the hospital during the same period and had no history of acute myocardial infarction and stroke were included as healthy control subjects. Exclusion criteria were: Aged $<18$ years old; suffering from cancer; pregnancy; patients with severe chronic diseases of the heart, liver, kidney or lung. In the present study, all experimental protocols were performed according to the approved guidelines and collected serum were immediately frozen in liquid nitrogen until further miR-186-5p analysis.

\section{Cell culture and OGD establishment and preparation}

SH-SY5Y cells were obtained from the American Type Culture Collection (Manassas, VA, USA). The cells were cultured in Dulbecco's Modified Eagle's Medium (DMEM) (HyClone, Logan, UT, USA) supplemented with $10 \%$ fetal bovine serum (Biological Industries, Beit-Haemek, Israel) and incubated in a humidified atmosphere of $5 \% \mathrm{CO} 2$ at $37^{\circ} \mathrm{C}$. The SH-SY5Y cells were cultured in OGD conditions for $10 \mathrm{~h}$ to mimic cerebral ischemia in vitro. Briefly, the SH-SY5Y cells complete culture medium was changed to glucose-free DMEM medium, and the culture flask was placed in a sealed tank with $\mathrm{N}_{2} / \mathrm{CO}_{2} / \mathrm{O}_{2}(94 \% / 5 \% / 1 \%)$ for 30 min, resulting in an oxygen content of 2-3\%. SH-SY5Y cells were then washed three times with this medium and incubated for $10 \mathrm{~h}$ in an oxygen-free $\mathrm{N}_{2} / \mathrm{CO}_{2} / \mathrm{O}_{2}(94 \% / 5 \% / 1 \%)$ atmosphere. Thereafter, the medium was replaced by standard culture medium. Conditioned medium was collected at $0,12,24$ and $48 \mathrm{~h}$ after reoxygenation respectively for further studies. SH-SY5Y cells were harvested at $24 \mathrm{~h}$, for MTT, flow cytometry, western blot and TUNEL.

\section{Cell apoptosis by flow cytometry}

miR-186-mimics (5'- CAAAGAAUUCUCCUUU UGGGCU-3') and miR-186 inhibitor (5'- AGCCCAAA AGGAGAAUUCUUUG - $3^{\prime}$ ) that have been used in this study are products of synthetic chemistry. Cell apoptosis was analyzed by use of flow cytometry and double staining cells with Annexin $\mathrm{V}$ and propidium iodide (PI). After treatment with miR-NC,
miR-186-mimics, miR-186 inhibitor, pCMV6 empty vector, pCMV6 + IGF-1, miR-NC, miR-186-mimisc + pCMV6-empty or miR-186-mimisc + pCMV6-IGF-1 in $\mathrm{OGD} / \mathrm{R}$, the neurons plated in 6-well cell culture cluster were washed twice with PBS and subjected to Annexin V-FITC/PI double staining at room temperature in the dark as described in the manufacturer's instructions (Annexin V-FITC Apoptosis Detection Kit (BD Biosciences, San Jose, $\mathrm{CA})$ ). After $15 \mathrm{~min}$ of staining, the neurons were analyzed by flow cytometry (BD Bioscience, San Jose, $\mathrm{CA})$ and the apoptotic rate was determined.

\section{Real-time polymerase chain reaction (PCR) analysis}

Real-time PCR was used to measure mRNA level of miR-186-5p and IGF-1, besides miR-186 was quantified by TaqMan probe (ID 002285) according to the instruction and operation manual. Total RNA was extracted using the TRIzol reagent (Thermo Fisher Scientific, Waltham, MA, USA) from tissues and cells. First-strand cDNA was synthesized using a reverse transcriptase kit (Applied Biosystems, Foster City, CA, USA) according to the manufacturer's instructions. Real-time PCRs were carried out with a SYBR Green PCR Master Mix Kit (Applied Biosystems) and performed on a 7500 FAST Real-Time PCR System (Applied Biosystems). GAPDH was used as an internal control. The following primers were used in this study. miR-186-5p; Forward, 5'- AAGAATTCTC CTTTTGGGCT -3', Reverse, 5' - GTGCGTGTCGTGGA GTCG -3'; IGF-1; Forward, 5' - TGTGTGGAGACAGG GGCTTT - 3 ', Reverse, 5' - TTGGCAGGCTTGAGGGG T -3'; GAPDH; Forward, 5'-ATCACTGCCACCCAGA AGAC3', Reverse, 5' -TTTCTAGACGGCAGGTCAGG $-3^{\prime}$.

\section{Western blot analysis}

The concentration of protein samples extracted from tissues and cells was determined using the BCA Protein Assay kit (Bio-Rad, Mississauga, ON, Canada). Protein samples were separated with $12 \%$ sodium dodecyl sulfate-polyacrylamide gel electrophoresis (SDS-PAGE) gel and transferred onto polyvinylidene fluoride (PVDF) membranes. After blocking in 5\% nonfat milk for 2 hours at room temperature, the membranes were treated with antibodies against caspase 3, cleaved caspase 3 and IGF-1 (Cell Signaling Technologies) and GAPDH (Santa Cruz Biotechnology, Santa Cruz, CA, USA) overnight at $48^{\circ} \mathrm{C}$, followed by secondary antibodies at room temperature for 1 hour. Western blot bands were quantified using Odyssey v1.2 software by measuring the band intensity (area $\times$ OD) for each group. GAPDH served as an internal control. 


\section{MTT assay}

Cell viability was determined by MTT assay according to the manufacturer's instructions. Briefly, cells $\left(2 \times 10^{4}\right.$ cells/well $)$ were seeded in a 96-well plate and treated with miR-NC, miR-186-mimics, miR-186 inhibitor, pCMV6 empty vector, pCMV6 + IGF-1, miR-186-mimisc + pCMV6-empty or miR-186-mimisc + pCMV6-IGF-1 in OGD/R. After treatment, $20 \mu \mathrm{L}$ of MTT solution $(0.5 \mathrm{mg} / \mathrm{mL})$ (Sigma Aldrich Co.) was added to each well and incubated at $37^{\circ} \mathrm{C}$ for 48 hours. After carefully removing the culture medium, $200 \mu \mathrm{L}$ of dimethyl sulfoxide (DMSO) was added to each well to dissolve the formazine granular, and the absorbance at $490 \mathrm{~nm}$ was measured using a microplate reader.

\section{Terminal deoxynucleotidyl transferase (TdT)-mediated-digoxigenin-1 1-dUTP nick end labeling (TUNEL).}

TUNEL was used to detect DNA fragmentation in SH-SY5Y cells treated with miR-NC, miR-186mimics or miR-186 inhibitor in OGD/R according to the instructions of TUNEL kit.

\section{Enzyme-linked Immunosorbent Assay (ELISA)}

The levels of IGF-1was measured by ELISA kits (70-EK11312, Kelian Bio-Tech, Hangzhou, China) according to the manufacturer's protocols. The intraand inter-assay CV are less $10 \%$.

\section{Luciferase assay}

Both IGF-1 $3^{\prime}$-UTRs containing conserved miR-186-5p binding sites and the mutated 3'-UTRs were synthesized by RIBOBIO (Guangzhou, China), and amplified by PCR. The 3'-UTR luciferase vector was cotransfected with miR-186-5p mimics into HEK293 cells using Lipofectamine 2000 (Invitrogen) and Renilla luciferase reporters were used as internal control. A luciferase activity assay was performed after $48 \mathrm{~h}$ using the Dual-Luciferase Reporter Assay System (Promega Biotech Co., Ltd.) according to the manufacturer's instructions.

\section{Statistical analysis}

Data are expressed as the mean \pm standard error of mean (SEM), and analyzed with SPSS 13.0 software (IBM Corporation, Armonk, NY, USA). Differences between two groups were examined using Student's t-test. Differences among multiple groups were evaluated using analysis of variance (ANOVA). A two-tailed $\mathrm{P}<0.05$ was considered as statistically significant.

\section{Results}

\section{IntracellularmiR-186-5p mRNA expression in OGD/R model}

GEO analysis indicated potential miRNA networks and target genes and we found obvious expression changes of hsa-miR-30a-5p, hsa-miR-30d5p, hsa-miR-185-5p, hsa-miR-186-5p, hsa-miR-324-5p, hsa-miR-342-3p and hsa-miR-361-5p in OGD10h/R model, among which hsa-miR-186-5p was the most significant. Besides, studies have shown that miR-186-5 $p$ has a close relationship with CNS diseases and ischemic diseases. Therefore, we focus the role miR-186-5p plays in stroke. Firstly, we use SH-SY5Y cells to build an OGD/R model in vitro to mimic ischemia and reperfusion. As shown in Figure 1, mRNA level of intracellular miR-186-5p was dramatically higher than that of control group in OGD10h/R model. Besides, the expression of miR-186-5p increased gradually and reached its peak at $24 \mathrm{~h}$. Other studies have found that OGD10h inducing SH-SY5Y cell apoptosis is also most obvious at R24 h. Taken together, we make a bold hypothesis that miR-186-5p might be associated with apoptosis in ischemia and reperfusion.

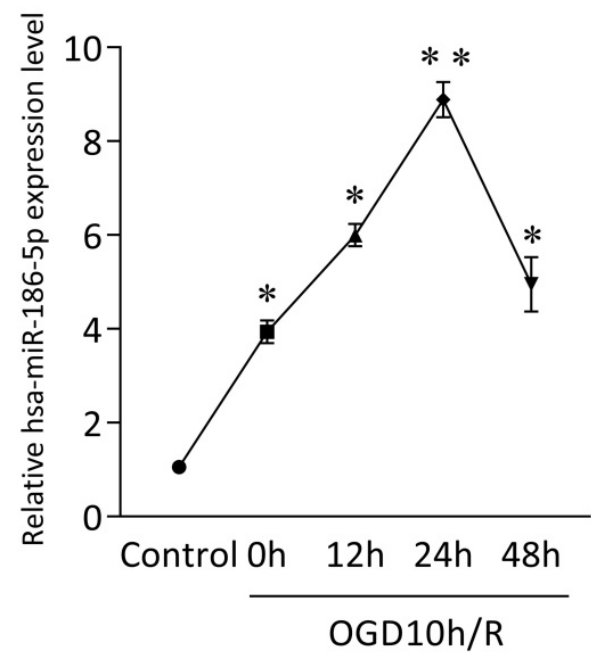

Figure 1. Change in intracellular miR-186-5p level in SH-SY5Y cells OGD10h/R model at the different time points, detected by qRT-PCR. The level of intracellular miR-186-5p was significantly increased, and reached its peak at $24 \mathrm{~h}$. $* * p<0.01$ versus control group; results are representative of three separate independent experiments.

\section{miR-186-5p had no effect on apoptosis on normal SH-SY5Y cells}

SH-SY5Y cells were treated with miR-NC, miR-186-mimics or miR-186-inhibitor. As expected, miR-186 mimics and inhibitor had a strong promoting or suppression effect on miR-186-5p mRNA expression (Figure 2A). Then cell apoptosis was measured by flow cytometry using Annexin V-FITC 

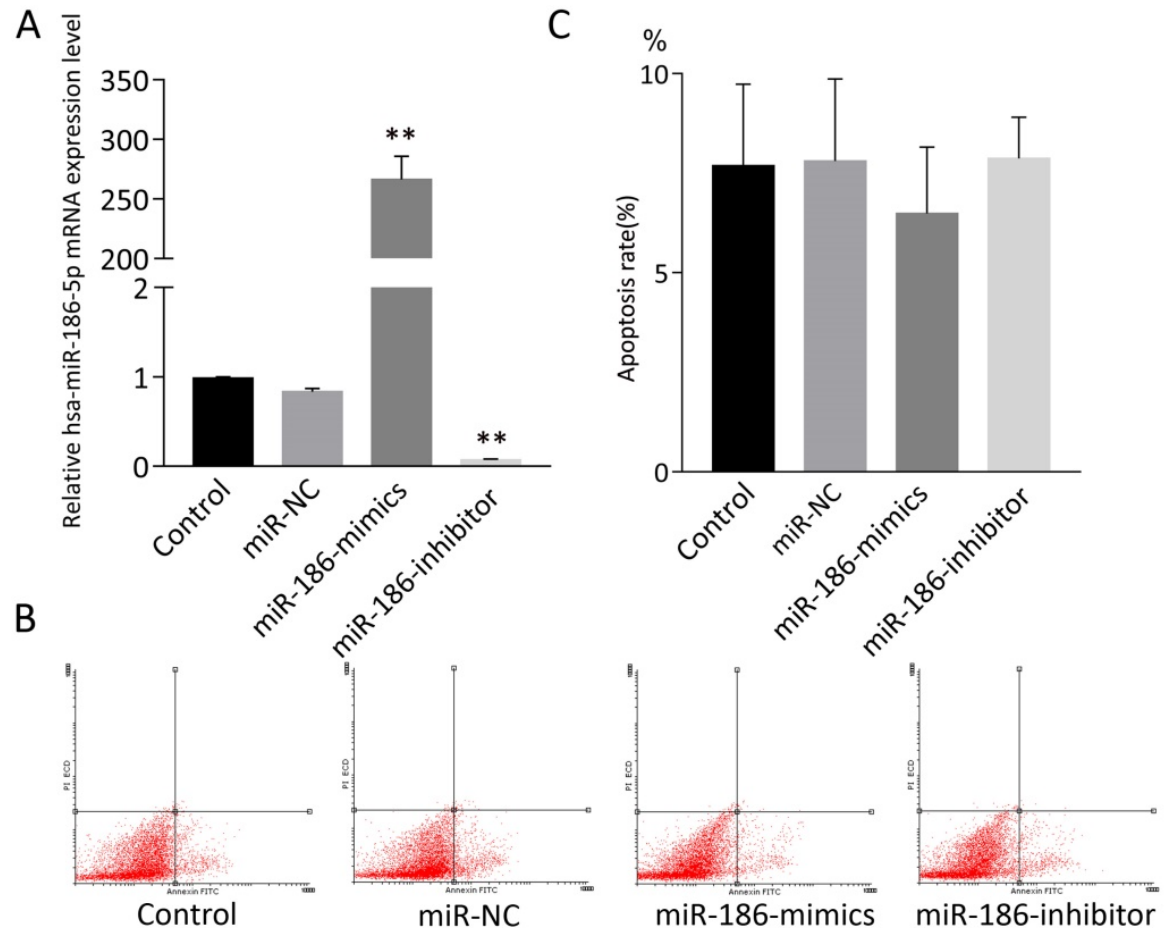

Figure 2. The effect of miR-186-5p on the apoptotic rate of SH-SY5Y cells. (A) qRT-PCRs were conducted to detect miR-186-5p mRNA expression under normal condition or with different treatment. (B) Annexin V-FITC/PI double-staining cells were examined by flow cytometry. (C) Analysis of apoptotic rate. $* * P<0.01$ versus control group; results are representative of three separate independent experiments. reduced in the groups treated with inhibitor targeting miR-186-5p (Figure 3D). Cleaved caspase 3 , the active form of caspase 3 , is an acknowledged marker of apoptosis. We then test the changes of different forms of caspase 3 in protein level by western blot. The variation trend was the same as the above (Figure 3E). OGD10h/R24h caused an increasing caspase 3 protein expression, meanwhile mimics and inhibitor had a positive or negative effective independently. So was the cleaved caspase 3 but there was nearly no cleaved caspase 3 expression in normal SH-SY5Y group. These results suggested miR-186-5p was upregulated in OGD10h/R and apoptosis was promoted by miR-186-5p only in OGD10h/R condition. However, miR-186-5p had no influence on apoptosis in normal SH-SY5Y cells.

and PI double staining. However, according to flow cytometry analysis, both miR-186 mimics and inhibitor could not influence cell apoptosis compared with control and miR-NC groups (Figure 2B, C).

\section{miR-186-5p reduced viability and motility of SH-SY5Y cells by inducing apoptosis}

But in OGD10h/R24h model, the results were quite different. As demonstrated by MTT assay, reperfusion led to a reduction of SH-SY5Y cell viability. miR-186-mimics showed a more significant inhibition on cells and miR-186-inhibitor reversed this suppression effect (Figure 3A). In flow cytometry assay, apoptosis rate was consistent. A stronger activation of apoptosis was observed in miR-186mimics group than that of control and miR-NC groups. While it was reduced by miR-186 inhibitor (Figure 3B, C). As we all known, apoptosis shows positive terminal deoxynucleotidyl transferasemediated dUTP nick end-labeling (TUNEL) staining in apoptotic cells death. Figure 3D showed the fluorescent signal of TUNEL and DAPI staining of SH-SY5Y cells in OGD10h/R24h model: TUNEL staining to monitor DNA damage, and DAPI staining to monitor morphological changes of nuclei. In the non-treated or miR-NC groups, there was a small number of dead cells. The cell death rate was significantly increased at $24 \mathrm{~h}$ after treated with miR-186 mimics. However, the increase was markedly

\section{miR-186-5p negatively modulates IGF-I expression}

We further explored the mechanism of how miR-186-5p enhanced apoptosis in OGD/R. TargetScan predicted that Insulin-like growth factor (IGF)-1 was of miR-16-5p biological targets. After treated with OGD10h/R24h, there was a remarkable overexpression of IGF-1 mRNA in SH-SY5Y cells. At the same time, miRNA-186-minics suppressed while miRNA-186-inhibitor enhanced IGF-1 mRNA expression (Figure 4A). As to protein level, same results were observed through western blot and ELISA assays (Figure 4B, C).

\section{IGF-1 suppressed miR-186-5p inducing cell apoptosis}

To further explore the effects of miR-186-5p and IGF-1 on cell apoptosis, we constructed sh-IFG-1. As shown in Fig 5A and 5B, there were obvious higher expression of IGF-1 on both mRNA and protein level after cells were transfected with sh-IFG-1. Then we tested cell apoptosis rate by flow cytometry, similar to Fig 3, miR-186-mimic up-regulated total apoptosis rate in OGD10h/R24h model, but this effect was reversed by IFG-1(Fig 5C, D). In TUNEL assay, as expected, IGF-1 could also inhibit apoptosis induced by miR-186-5p (Fig 5E). 


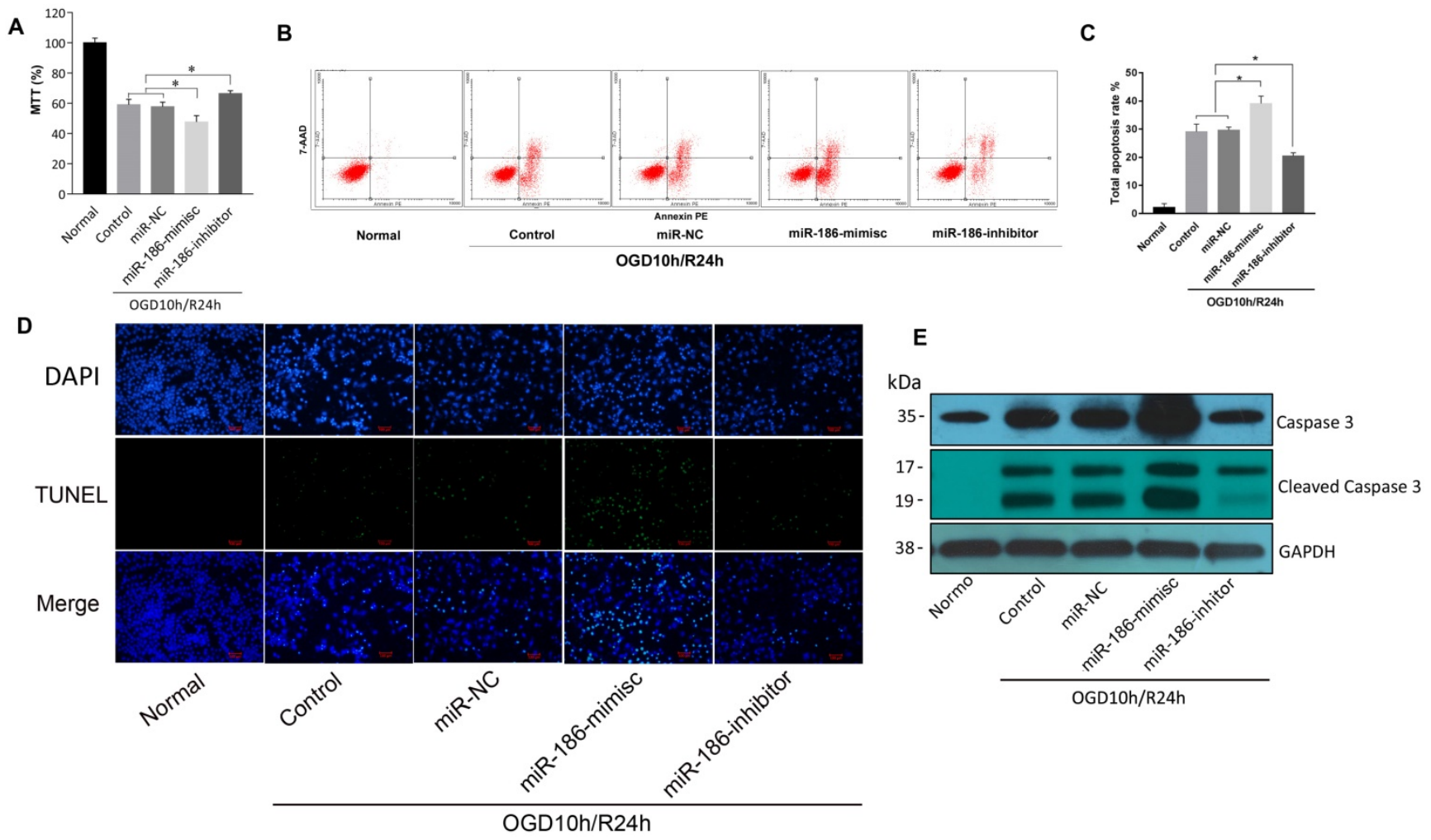

Figure 3. The effect of miR-186-5p on the apoptotic rate of the OGD/R-treated SH-SY5Y cell. The SH-SY5Y cells were treated with miR-NC, miR-186-mimics or miR-186-inhibitor. (A) MTTs were conducted to detect SH-SY5Y cell viability in OGD/R model with different treatment. (B) Annexin V-FITC/PI double-staining cells were examined by flow cytometry. (C) Analysis of apoptotic rate. Cell apoptosis was analyzed by use of flow cytometry and double staining cells with Annexin $V$-FITC and propidium iodide. (D) SH-SY5Y cells in OGD/R group were treated with miR-NC, miR-186-mimics or miR-186 inhibitor for 48 h. A representative image of TUNEL assay was shown. TUNEL - positive cells, green; nuclei, blue. Scale bar: $100 \mu \mathrm{m}$. (E) Western blots were performed to detect the expression of Caspase 3 and cleaved Caspase 3. GAPDH was used as internal control. The bands were shown. $* P<0.05$, $* * P<0.01$ versus control or miR-NC group; results are representative of three separate independent experiments.

\section{Direct targeting effect of miR-186-5p on the 3' UTR of IGF-I}

Previous study showed that miR-185-5p played an important role in ischemia and reperfusion, by means of testing miR-185-5p's affection on IGF-1, we predicted that miR-186-5p was involved in the interaction between IGF-1 and apoptosis. Analyses indicated a direct targeting effect of miR-186-5p on the 3' UTR of IGF-1 (Figure 6A). We cloned the 3' UTR of 3 wild types of IGF-1 mRNA into a luciferase reporter vector and transfected the cloned plasmid with either scrambled miRNA or miR-186-5p into SH-SY5Y cells. The plasmids were WT-1 (Position 1903-1909 of IGF1 3' UTR), WT-3 (Position 3676-3682 and 4184-4190 of IGF1 3' UTR) and WT-2 (Position 6594-6600 of IGF1 3' UTR). MiR-186-5p did not suppress the luciferase activity of the reporter vector containing 3' UTR of IGF-1-WT1 and WT2 with single-point mutations in the miR-186-5p-binding site (Figure 6B). But miR-186-5p could significantly inhibit the luciferase activity of IGF-1-WT3 containing two mutations compared with the others. Then, another three WT3 plasmids were built, including (3676-3682 and 4184-4190 co-mutation), MT-2 (3676-3682 mutation,
4184-4190 wild type) and MT-3 (4184-4190 mutation, 3676-3682 wild type). As shown in Figure 6C, only MT-3 had a significance difference, suggesting that 3676-3682 was miR-186-5p binding site and miR-186-5p could directly interact with IGF-1.

\section{miR-186-5p was upregulated in ischemic stroke patients' serum}

To confirm our finding in ischemic stroke patients, we studied miR-186-5p serum concentration in 23 healthy and 23 patients. The median age of patients included in this study was 65 (IQR, 44-83) years and $52.2 \%$ were men. The median NIHSS score on admission was 11 points (IQR, 2 to 16). As expected, serum miR-186-5p level was higher in ischemic stroke patients as compared to control group $(P=0.029$; Figure 7.), which had the same trend in OGD/R model experiments.

\section{Discussion}

Ischemic stroke, which leads to severe disability in adults, makes up approximately $87 \%$ of all stroke cases and has been intensively studied[7, 8]. However, effective therapies are limited by several reasons, such as the complex interaction among different signal 
pathways, the rapid development of brain injury after ischemia, the appropriate treatment window for specific targets[1,9]. Even though large amount of efforts was spent on preclinical trials targeting agents that involved in the pathophysiologic mechanisms, results were far from satisfactory. In recent years, accumulating evidence has demonstrated that miRNAs have also neuroprotective roles in ischemic stroke [9-11].

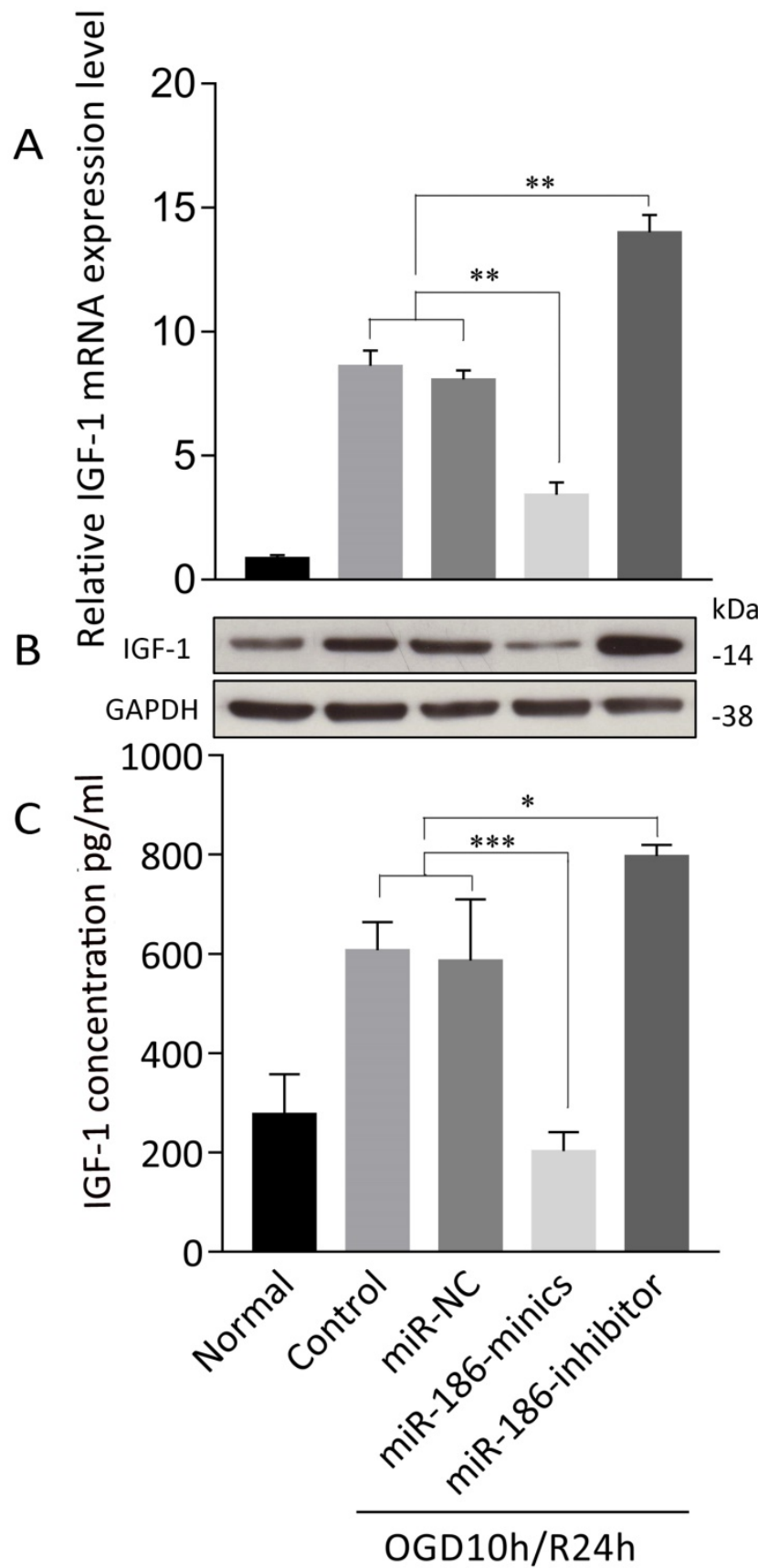

Figure 4. miR-186-5p affected IGF-1 expression in OGD/R-treated SH-SY5Y cell. SH-SY5Y cells in OGD/R group were treated with miR-NC, miR-186-mimics or miR-186 inhibitor. (A) IGF-1 mRNA expression was detected by qRT-PCR. (B) Tthe protein expression of IGF-1 was analyzed by western blots. GAPDH was used as internal control. (C) IFG-1 was quantified by ELISA. $* P<0.05, * * P<0.01, * * * P<0.001$ versus control or miR-NC group; results are representative of three separate independent experiments.
miR-186-5p has been found to play dual roles either as an oncogene or a tumor suppresser gene in several kinds of cancers[12]. For example, in NSCLC cells, miR-186-5p suppresses tumor proliferation by regulating cyclin D1, CDK2 and CDK6, and miR-186-5p downregulation is correlated with poor survival[13]. On the contrary, miR-186-5p also acts as an oncogene to promote endometrial tumorigenesis by inhibiting the expression of tumor suppressor gene FOXO1[14]. Besides, miR-186-5p is previously reported to serve as a circulating biomarker of cardiovascular risk and facilitate the diagnosis of acute coronary syndrome[6, 15]. As reported, blood-brain barrier (BBB) was destroyed and its permeability was enhanced in ischemic stroke[16, 17]. Thus, more small molecule materials can go through BBB and go into blood circulation. Therefore, miR-186-5p in serum reflects its concentration in central nervous system. In this study, we found that miR-186-5p was upregulated dramatically both in OGD/R model and ischemic stroke patients' serum. It may be a potential serum diagnostic marker of ischemic stroke. Other researches prove that miR-186-5p can modulate apoptosis in a certain type of human cells[18, 19].There was also an obvious apoptosis induced by miR-186-5p in OGD/R SH-SY5Y cells, demonstrated by our in vitro experiments.

IGF-1, regulated by growth hormone, is present in many cell types, including neurons and glia [20]. It is essential for the development of the nervous system, hippocampal neurogenesis, and neurotransmission [21]. IGF-1 regulates cell growth, differentiation and survival of neuronal cell lines, and is one of the most potent anti-apoptotic growth factors present in eukaryotic cells[22, 23]. IGF-1 has a neuroprotective effect in stroke animal model, and high serum IGF-1 levels just after ischemic stroke onset are associated with better neurological recovery and functional outcome, suggesting that the endogenous IGF-1 level impacts on the evolution of cerebral infarction [24, 25].

The blood-brain barrier (BBB) is a dynamic interface between brain compartments and blood vessels that can efficiently protect nerves from injury and infection. But an increase of permeability of BBB was observed after cerebral ischemia, and there are even some new administration methods that deliver drugs systemically depend on the breakdown of $\mathrm{BBB}[26]$. There is increasing evidence showing microRNAs can cross BBB because of ischemia-induced cerebral endothelial injury and BBB leakage[27, 28]. Therefore, microRNAs can be found in circulatory biofluids, such as blood and blood components, plasma and serum. Blood and blood 
components are primary sources where miRNAs are released in either an encapsulated form with microvesicle particles, such as exosome, or are in cell-free form and then bind to protein components[29]. In further research, we will explore what is the major format of miR-186-5p in serum and how it binds to or taken up by neurons in brain. Besides, we will also try to find more genes and pathways regulated by miR-186-5p in ischemic stroke.
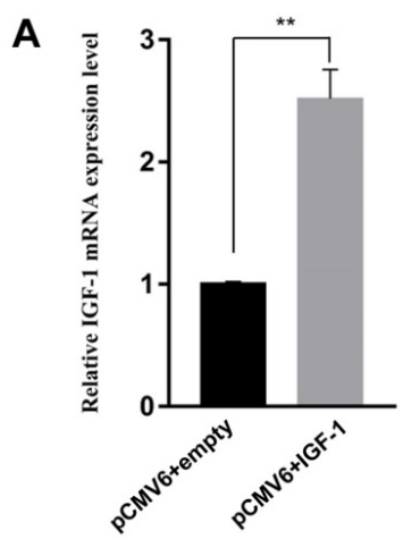

B

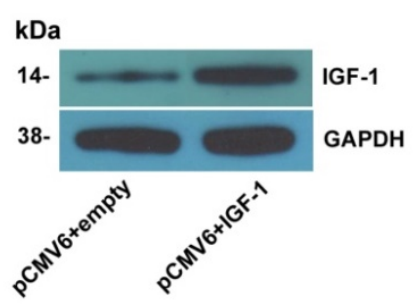

C

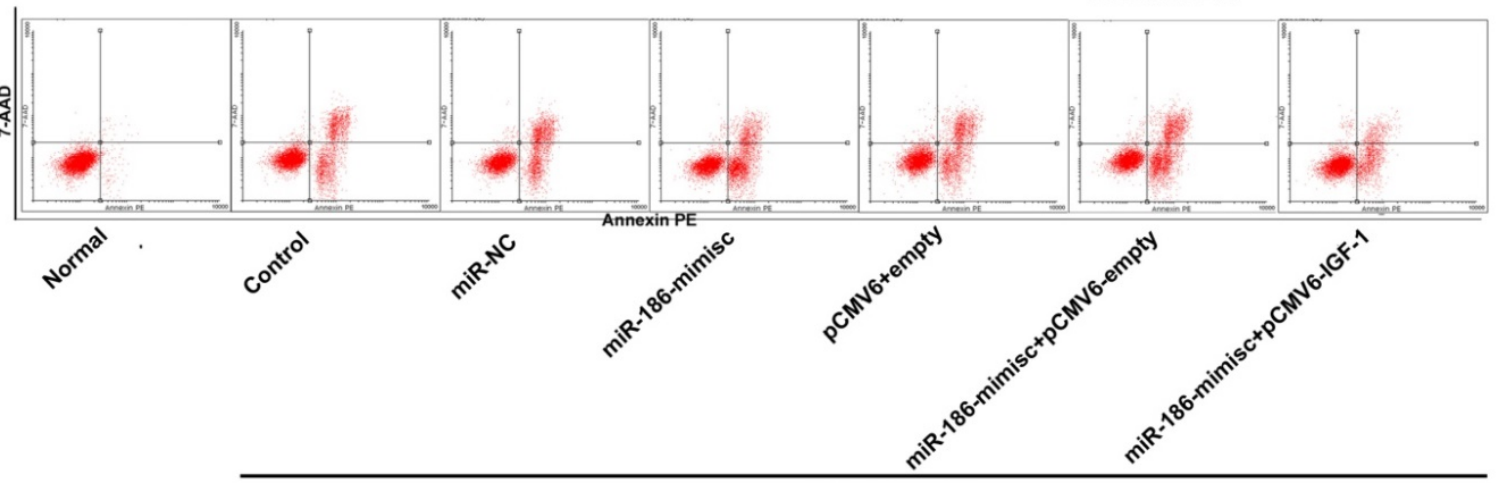

OGD10h/R24

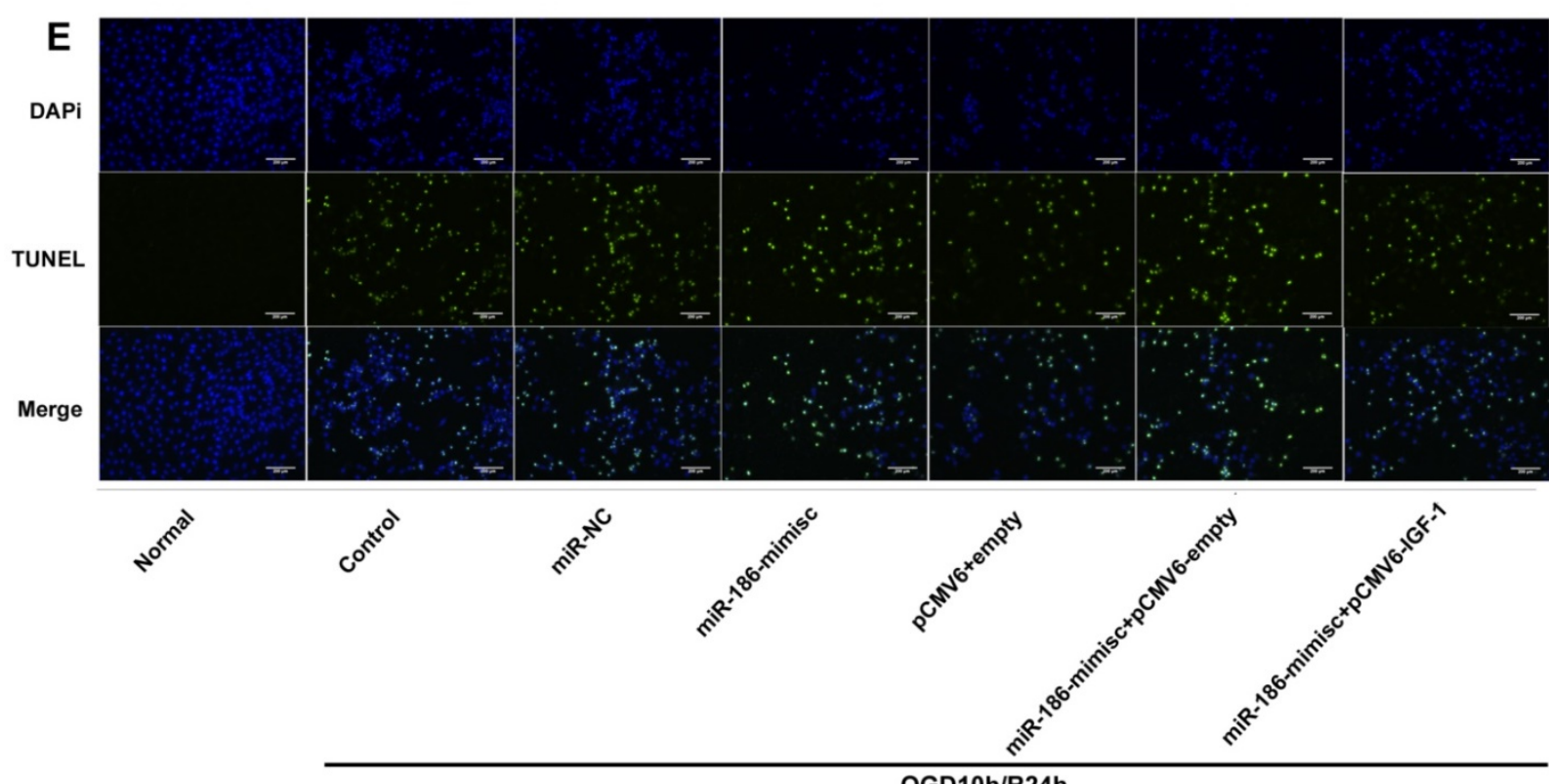

OGD10h/R24h

Figure 5. IGF-1 reduced miR-186-5p inducing cell apoptosis. SH-SY5Y cells were transfected with the pCMV6 empty vector, pCMV6 + IGF-1, miR-NC, miR-186-mimisc, miR-186-mimisc + pCMV6-empty or miR-186-mimisc + pCMV6-IGF-1 in normal or OGD10h/R24 conditions. (A) IGF-1 mRNA expression was detected by qRT-PCR. (B) The protein expression of IGF-1 was analyzed by western blots. GAPDH was used as internal control. (C) Annexin V-FITC/PI double-staining cells in different treatment groups were examined by flow cytometry. (D) Analysis of apoptotic rate. (E) A representative image of TUNEL assay was shown. TUNEL - positive cells, green; nuclei, blue. Scale bar: $100 \mu \mathrm{m}$. $* p<0.05$, $* * p<0.01$ versus $p C M V 6+$ empty, miR-NC or control or miR-186-mimisc + pCMV6-empty group; results are representative of three separate independent experiments. 

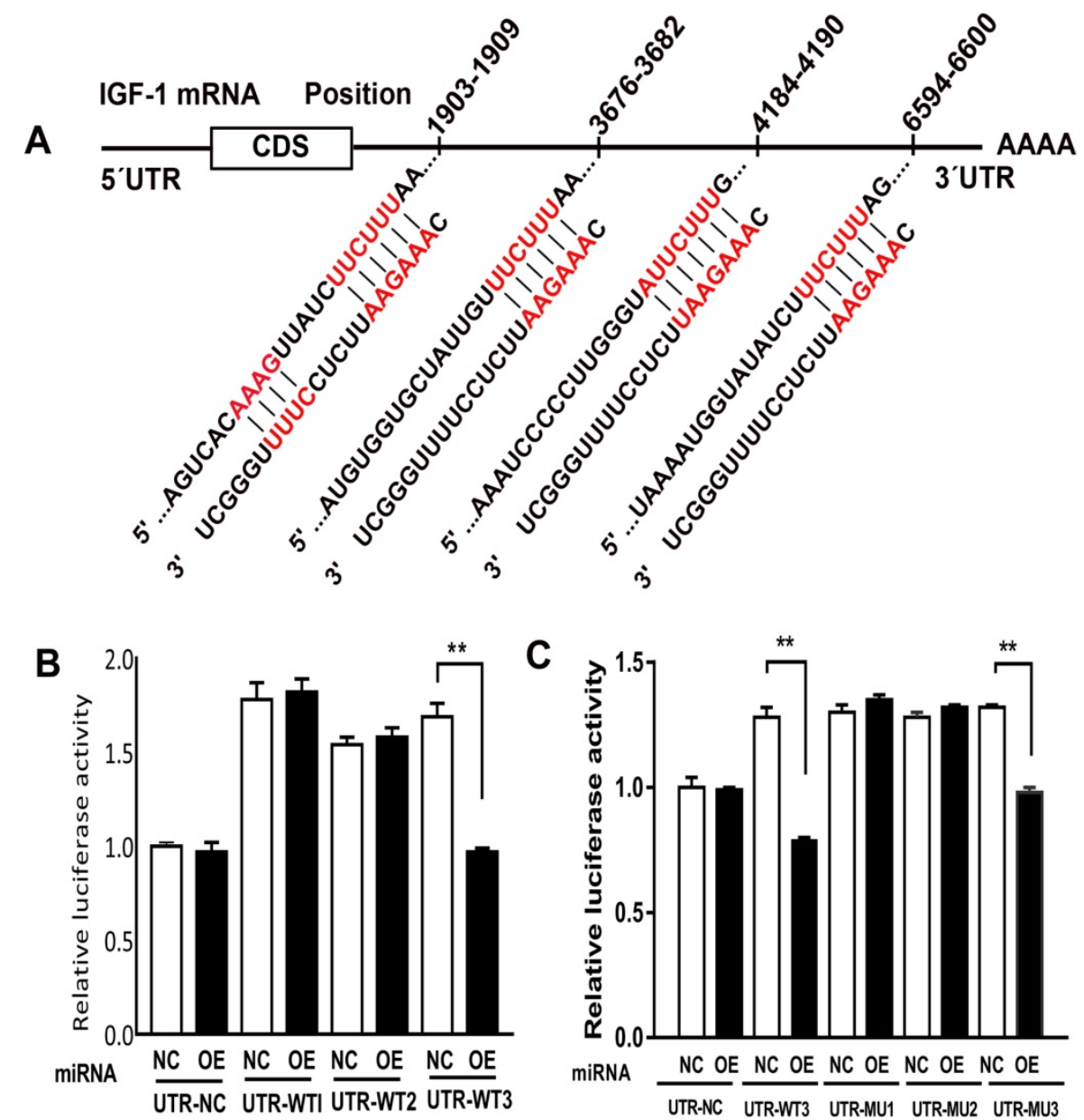

Figure 6. miR-186-5p regulated IGF-1. (A) Predicted consequential pairing of target region and miRNA. (B, C) Luciferase report assay was performed to detect the interaction between miR-186-5p and IGF-1. **P $<0.01$ versus NC group; results are representative of three separate independent experiments.

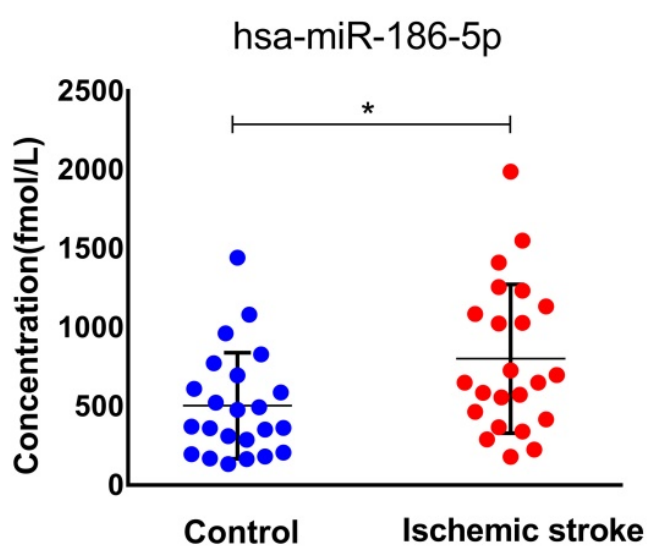

Figure 7. Serum miR-186-5p levels in acute ischemic stroke patients and control group. Significantly higher in stroke patients as compared to control cases $(P=0.029)$; results are representative of three separate independent experiments.

Here, we found miR-186-5p could directly suppressed IGF-1 expression and induced remarkable apoptosis of neurons, which meant that miR-186-5p played a harmful role in ischemic stroke. But,
miR-186-5p inhibitor could eliminate this negative effect and might be a good drug for the therapy of stroke by inhibiting neurons' apoptosis.

\section{Acknowledgments}

This work was supported by grant National Natural Science Foundation of China (No.81372173, 81572743) and Natural Science Foundation of Heilongjiang Province (D201202).

\section{Competing Interests}

The authors have declared that no competing interest exists.

\section{References}

1. Zhang $X$, Tang $X$, Liu K, Hamblin MH, Yin KJ. Long Noncoding RNA Malat1 Regulates Cerebrovascular Pathologies in Ischemic Stroke. Journal of Neuroscience the Official Journal of the Society for Neuroscience. 2017; 37: 1797-806.

2. Liu B, Zhang $Y H$, Jiang $Y$, Li LL, Chen $\mathrm{O}, \mathrm{He}$ GQ, et al. Gadd45b is a novel mediator of neuronal apoptosis in ischemic stroke. International journal of biological sciences. 2015; 11: 353-60.

3. Iorio MV, Croce CM. microRNA involvement in human cancer. Carcinogenesis. 2012; 33: 1126-33.

4. Wu G, Liu J, Wu Z, Wu X, Yao X. MicroRNA-184 inhibits cell proliferation and metastasis in human colorectal cancer by directly targeting IGF-1R. Oncology letters. 2017; 14: 3215-22. 
5. Bartel DP. MicroRNAs: genomics, biogenesis, mechanism, and function. Cell. 2004; 116: 281-97.

6. Wang KJ, Zhao X, Liu YZ, Zeng QT, Mao XB, Li SN, et al. Circulating MiR-19b-3p, MiR-134-5p and MiR-186-5p are Promising Novel Biomarkers for Early Diagnosis of Acute Myocardial Infarction. Cellular physiology and biochemistry : international journal of experimental cellular physiology, biochemistry, and pharmacology. 2016; 38: 1015-29.

7. Wu Z, Wu P, Zuo $X, Y u$ N, Qin $Y, X u$ Q, et al. LncRNA-N1LR Enhances Neuroprotection Against Ischemic Stroke Probably by Inhibiting p53 Phosphorylation. Molecular neurobiology. 2017; 54: 7670-85.

8. Mozaffarian D, Benjamin EJ, Go AS, Arnett DK, Blaha MJ, Cushman M, et al. Heart Disease and Stroke Statistics-2016 Update: A Report From the American Heart Association. Circulation. 2016; 133: e38-360.

9. Yin KJ, Deng Z, Huang H, Hamblin M, Xie C, Zhang J, et al. miR-497 regulates neuronal death in mouse brain after transient focal cerebral ischemia. Neurobiology of disease. 2010; 38: 17-26.

10. Yin KJ, Deng Z, Hamblin M, Xiang Y, Huang H, Zhang J, et al. Peroxisome proliferator-activated receptor delta regulation of miR-15a in ischemia-induced cerebral vascular endothelial injury. The Journal of neuroscience : the official journal of the Society for Neuroscience. 2010; 30: 6398-408.

11. Harraz MM, Eacker SM, Wang X, Dawson TM, Dawson VL. MicroRNA-223 is neuroprotective by targeting glutamate receptors. Proceedings of the National Academy of Sciences of the United States of America. 2012; 109: 18962-7.

12. Zhao X, Wang Y, Deng R, Zhang H, Dou J, Yuan H, et al. miR186 suppresses prostate cancer progression by targeting Twist1. Oncotarget. 2016; 7: 33136-51.

13. Cai J, Wu J, Zhang H, Fang L, Huang Y, Yang Y, et al. miR-186 downregulation correlates with poor survival in lung adenocarcinoma, where it interferes with cell-cycle regulation. Cancer research. 2013; 73: 756-66.

14. Myatt SS, Wang J, Monteiro LJ, Christian M, Ho KK, Fusi L, et al. Definition of microRNAs that repress expression of the tumor suppressor gene FOXO1 in endometrial cancer. Cancer research. 2010; 70: 367-77.

15. Wu J, Song J, Wang C, Niu D, Li H, Liu Y, et al. Identification of serum microRNAs for cardiovascular risk stratification in dyslipidemia subjects. International journal of cardiology. 2014; 172: 232-4.

16. Liu YC, Tsai YH, Tang SC, Liou HC, Kang KH, Liou HH, et al. Cytokine MIF Enhances Blood-Brain Barrier Permeability: Impact for Therapy in Ischemic Stroke. Scientific reports. 2018; 8: 743.

17. O'Donnell ME. Blood-brain barrier $\mathrm{Na}$ transporters in ischemic stroke. Advances in pharmacology (San Diego, Calif). 2014; 71: 113-46.

18. He W, Feng J, Zhang Y, Wang Y, Zang W, Zhao G. microRNA-186 inhibits cell proliferation and induces apoptosis in human esophageal squamous cell carcinoma by targeting SKP2. Laboratory investigation; a journal of technical methods and pathology. 2016; 96: 317-24.

19. Zheng $\mathrm{J}, \mathrm{Li} X \mathrm{X}$, Wang $\mathrm{P}$, Liu XB, Xue $\mathrm{YX}, \mathrm{Hu} \mathrm{Y}$, et al. CRNDE affects the malignant biological characteristics of human glioma stem cells by negatively regulating miR-186. Oncotarget. 2015; 6: 25339-55.

20. Bondy $\mathrm{C}$, Werner $\mathrm{H}$, Roberts $\mathrm{CT}$, Jr, LeRoith $\mathrm{D}$, Cellular pattern of type-I insulin-like growth factor receptor gene expression during maturation of the rat brain: comparison with insulin-like growth factors I and II. Neuroscience. 1992; 46: 909-23.

21. D'Ercole AJ, Ye P, Calikoglu AS, Gutierrez-Ospina G. The role of the insulin-like growth factors in the central nervous system. Molecular neurobiology. 1996; 13: 227-55.

22. Seto $\mathrm{D}$, Zheng $\mathrm{WH}$, McNicoll $\mathrm{A}$, Collier $\mathrm{B}$, Quirion $\mathrm{R}$, Kar $\mathrm{S}$. Insulin-like growth factor-I inhibits endogenous acetylcholine release from the rat hippocampal formation: possible involvement of GABA in mediating the effects. Neuroscience. 2002; 115: 603-12.

23. Trejo JL, Piriz J, Llorens-Martin MV, Fernandez AM, Bolos M, LeRoith D, et al. Central actions of liver-derived insulin-like growth factor I underlying its pro-cognitive effects. Molecular psychiatry. 2007; 12: 1118-28.

24. De Smedt A, Brouns R, Uyttenboogaart M, De Raedt S, Moens M, Wilczak N, et al. Insulin-like growth factor I serum levels influence ischemic stroke outcome. Stroke. 2011; 42: 2180-5

25. Wang J, Tang Y, Zhang W, Zhao H, Wang R, Yan Y, et al. Insulin-like growth factor-1 secreted by brain microvascular endothelial cells attenuates neuron injury upon ischemia. The FEBS journal. 2013; 280: 3658-68.

26. Fletcher L, Isgor E, Sprague S, Williams LH, Alajajian BB, Jimenez DF, et al. Spatial distribution of insulin-like growth factor binding protein-2 following hypoxic-ischemic injury. BMC neuroscience. 2013; 14: 158

27. Yin KJ, Hamblin M, Chen YE. Non-coding RNAs in cerebral endothelial pathophysiology: emerging roles in stroke. Neurochemistry international. 2014; 77: 9-16.

28. Kalani A, Tyagi A, Tyagi N. Exosomes: mediators of neurodegeneration, neuroprotection and therapeutics. Molecular neurobiology. 2014; 49: 590-600.

29. Rink C, Khanna S. MicroRNA in ischemic stroke etiology and pathology. Physiological genomics. 2011; 43: 521-8. 